\title{
Liste der Abkürzungen
}

\section{Zeitschriften und Serien}

$A A D$

$A A L$

AArmL

$A A S$

$A A S t$

$A B G$

$A E$

AEASH

AEHE-HPh

$A F$

AFFB

AFLLSCF

AFMP

AFS

$A G H B$

AHASH

AIGK

AIUON

AIUON-S

AIUON-L

AIUON-O

AIsl

AJAMES

$A K u$

AKM

$A L$

ALASH

ALM

AmA

Anatolica

AnL

AnLing

ANU BiH

$A O$

$\mathrm{AOH}$
Akademik Araştırmalar Dergisi (İstanbul)

Asien Afrika Lateinamerika (Berlin)

Annual of Armenian Linguistics (Cleveland, $\mathrm{OH}$ )

Asian and African Studies (Bratislava)

Asian and African Studies (Jerusalem)

Ankaraner Beiträge zur Germanistik (Ankara)

Avrasya Etüdleri (Ankara)

Acta Ethnographica Academiae Scientiarium Hungaricae (Budapest)

Annuaire de l'École pratique des Hautes Études. IV ${ }^{\mathrm{e}}$ section:

Sciences historiques et philologiques (Paris)

Alanya Folkloru (Alanya)

Anali Filološkog Fakulteta/Annales de la Faculté de Philologie

(Beograd)

Annali della Facoltà di Lingue e Litterature Stranieri di Ca'Foscari

(Venezia-Brescia)

Annali della Facoltà di Magistero. Università di Palermo (Palermo)

Asian Folklore Studies (Nagoya)

Anali Gazi Husrev-begove biblioteke (Sarajevo)

Acta Historica Academiae Scientiarum Hungaricae (Budapest)

Akten des ... Internationalen Germanisten-Kongresses (Bern)

Annali dell'Istituto Universitario Orientale di Napoli (Napoli)

Annali dell'Istituto Universitario Orientale di Napoli.

Sezione slava (Napoli)

Annali dell'Istituto Universitario Orientale di Napoli.

Sezione linguistica (Napoli)

Annali dell'Istituto Universitario Orientale di Napoli. Rivista del

Dipartimento di Studi Asiatici e del Dipartimento di Studi e Ricerche su Africa e Paesi Arabi (Napoli)

Annales Islamologiques (Le Caire)

Annals of Japan Association for Middle East Studies (Tokyo)

Acta Kurdica. International Journal of Kurdish \& Iranian Studies

(London)

Atatürk Kültür Merkezi

Acta Linguistica Hafniensia. International Journal of General Linguistics

(Copenhagen)

Acta Linguistica Academiae Scientiarum Hungaricae (Budapest)

Atlante Linguistico Mediterraneo

American Anthropologist (Menasha, WI)

Anatolica. Annuaire international pour les civilisations de l'Asie antărieure (Leiden)

Anthropological Linguistics (Bloomington, IN)

Analecta Linguistica (Amsterdam)

Akademija Nauka i Umjetnosti Bosne i Hercegovine (Sarajevo)

Acta Orientalia (Copenhagen)

Acta Orientalia Academiae Scientiarum Hungaricae (Budapest) 


\begin{tabular}{|c|c|}
\hline$A P h W$ & Acta Philologica (Warszawa) \\
\hline AppL & Applied Linguistics (Oxford) \\
\hline AppP & $\begin{array}{l}\text { Applied Psycholinguistics. Psychological studies of language processes } \\
\text { (Cambridge) }\end{array}$ \\
\hline Aramco $W M$ & Aramco World Magazine (New York) \\
\hline Arayışlar & Aray1şlar (Isparta) \\
\hline ArchOtt & Archivum Ottomanicum (Wiesbaden) \\
\hline $\operatorname{Arch} P$ & 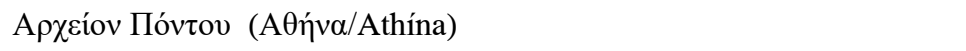 \\
\hline ArchThTh & 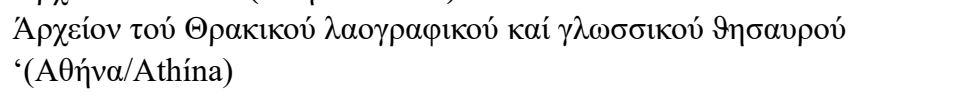 \\
\hline $\mathrm{ArOr}$ & Archiv Orientální (Praha) \\
\hline AsAfrL & Ajia Afurika bunpō kenkyū/Asian and African Linguistics (Tōkyō) \\
\hline$A S A L$ & American Studies in Altaic Linguistics (Bloomington, IN) \\
\hline ASEER & The American Slavic and East European Review (Cambridge, MA) \\
\hline$A S L$ & Annales. Sectio Linguistica (Budapest) \\
\hline$A S P h$ & Anzeiger für Slawische Philologie (Wiesbaden) \\
\hline$A S S C$ & The Annual of the Society for the Study of Caucasia (Chicago, IL) \\
\hline ASSEO SLF & $\begin{array}{l}\text { Annali del Seminario di Studi dell'Europa Orientale. } \\
\text { Sezione linguistico-filologica (Roma) }\end{array}$ \\
\hline$A \theta \eta v \alpha ́$ & 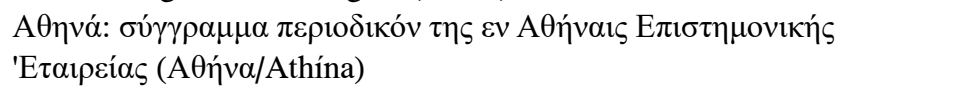 \\
\hline AÜ & Ankara Üniversitesi \\
\hline$A \ddot{U} B Y$ & Ankara Üniversitesi Basın-Yayın Yüksek Okulu Yıllı̆̆ı \\
\hline AÜDTCF & Ankara Üniversitesi Dil ve Tarih-Coğrafya Fakültesi \\
\hline$A \ddot{U} D T C F B D E D$ & $\begin{array}{l}\text { Ankara Üniversitesi Dil ve Tarih-Coğrafya Fakültesi Batı Dilleri ve } \\
\text { Edebiyatları Araştırma Enstitüsü Dergisi (Ankara) }\end{array}$ \\
\hline$A \ddot{U D T C F D}$ & Ankara Üniversitesi Dil ve Tarih-Coğrafya Fakültesi Dergisi (Ankara) \\
\hline AÜSBF & Ankara Üniversitesi Siyasal Bilgiler Fakültesi \\
\hline$A \ddot{U S B F D}$ & Ankara Üniversitesi Siyasal Bilgiler Fakültesi Dergisi (Ankara) \\
\hline AUSBREL & $\begin{array}{l}\text { Annales Universitatis Scientiarum Budapestinensis de Rolando Eötvös } \\
\text { nominatae, Sectio linguistica (Budapest) }\end{array}$ \\
\hline$A \ddot{U T A E D}$ & $\begin{array}{l}\text { Atatürk Üniversitesi Türkiyat Araştırmaları Enstitüsü Dergisi/Journal of } \\
\text { Turkish Research Institute (Erzurum) }\end{array}$ \\
\hline AVT & Algemene Vereniging voor Taalwetenschap/Dutch Linguistics Assoc. \\
\hline$A Y B$ & Azerbaycan Yurt Bilgisi (İstanbul) \\
\hline Ayre & Ayre. Mecmuaya Zıwanî (Stockholm) \\
\hline Avtoref. dis. & Avtoreferat dissertacii (=Abstract der Dissertation) \\
\hline Avtoref. dis. dokt. fil. & $\begin{array}{l}\text { Avtoreferat dissertacii na soiskanie učeoj stepeni doktora filologičeskih } \\
\text { nauk (=Abstract der Doktorarbeit) }\end{array}$ \\
\hline Avtoref. dis. kand. fil. & $\begin{array}{l}\text { Avtoreferat dissertacii na soiskanie učeoj stepeni kandidata } \\
\text { filologičeskih nauk (=Abstract der Kandidaturdissertation) }\end{array}$ \\
\hline Azärbajğan & Azärbajğan (Baku) \\
\hline$B A$ & Balkan-Archiv. Neue Folge (Gerbrunn bei Würzburg) \\
\hline Bağlam & $\begin{array}{l}\text { Bağlam. İstanbul Üniversitesi Yabancı Diller Yüksek Okulu Almanca } \\
\text { Bölümü Dergisi (İstanbul) }\end{array}$ \\
\hline Balcanica & $\begin{array}{l}\text { Balcanica. Annuaire de l'Institut des Études balkaniques, Academie } \\
\text { Serbe des Sciences et des Arts/Balkanika. Godišnjak Instituta za } \\
\text { Balkanologiju, Srpska Akademija Nauka i Umetnosti (Beograd) }\end{array}$ \\
\hline
\end{tabular}

$A P h W$

AppL

AppP

Aramco WM

Arayışla

ArchP

ArchThTh

ArOr

AsAfrL

$A S A L$

ASEER

$A S L$

$A S P h$

ASSC

ASSEO SLF

$A \theta \eta v \alpha ́$

AÜ

$A \ddot{U B Y}$

AÜDTCF

$A \ddot{U D T C F D}$

$\mathrm{AÜSBF}$

$A \ddot{U S B F D}$

$A Y B$

Ayre

Avtoref. dis.
Acta Philologica (Warszawa)

(Cambridge)

Arayışlar (Isparta)

Archivum Ottomanicum (Wiesbaden)

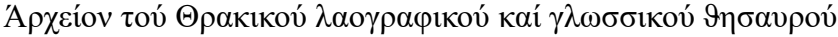

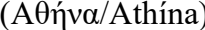

Ajia Afurika bunpō kenkyū/Asian and African Linguistics (Tōkyō)

American Studies in Altaic Linguistics (Bloomington, IN)

The American Slavic and East European Review (Cambridge, MA)

Annales. Sectio Linguistica (Budapest)

Anzeiger für Slawische Philologie (Wiesbaden)

The Annual of the Society for the Study of Caucasia (Chicago, IL)

Annali del Seminario di Studi dell'Europa Orientale.

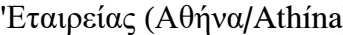

Ankara Üniversitesi Dil ve Tarih-Coğrafya Fakültesi

Ankara Üniversitesi Dil ve Tarih-Coğrafya Fakültesi Batı Dilleri ve

Edebiyatları Araştırma Enstitüsü Dergisi (Ankara)

Ankara Üniversitesi Dil ve Tarih-Coğrafya Fakültesi Dergisi (Ankara)

Ankara Üniversitesi Siyasal Bilgiler Fakültesi

Ankara Universitesi Siyasal Bilgiler Fakültesi Dergisi (Ankara)

nominatae, Sectio linguistica (Budapest)

Turkish Research Institute (Erzurum)

Azerbaycan Yurt Bilgisi (İstanbul)

Ayre. Mecmuaya Zıwanî (Stockholm)

Avtoreferat dissertacii (=Abstract der Dissertation)

nauk (=Abstract der Doktorarbeit)

Bakanologiju, Srpska Akademija Nauka i Umetnosti (Beograd) 
Balkanistica

Balkanistika

BalkE

BALM

BalSt

$B \& L$

$B C A I$

BCILA

$B E$

BEBS

Belleten

BFLS

BHR

BIG

Bilge

Bilig

$\mathrm{BiOr}$

Bir

$B J L$

BJMES

$B J R L$

BKart

BLR

BMGSt

BNam

$\mathrm{BOH}$

$B P P$

$B r L$

$B S$

$B S L P$

BSMESB

$B S O A S$

$B T T D$

$B \ddot{U} D$

$B \ddot{U} D B B$

$B \ddot{U} H Y$

BUT-ShSh

Byzantinoslavica

CAJ
Balkanistica. Occasional Papers in Southeast European Studies

(Columbus, $\mathrm{OH}$ )

Balkanistika (Sofija)

Balkansko Ezikoznanie/Linguistique Balkanique (Sofija)

Bolletino dell'Atlante Linguistico Mediterraneo (Venezia-Pisa)

Balkan Studies (Thessaloniki)

Brain and Language (New York)

Bulletin Critique des Annales Islamologiques (La Caire)

Bulletin de la Comission Interuniversitaire Suisse de Linguistique

Appliquée (Neuchâtel, $\mathrm{CH}$ )

Bălgarski Ezik (Sofija)

Bochum-Essener Beiträge zur Sprachwandelforschung (Bochum)

Belleten (Ankara)

Bulletin de la Faculté des Lettres de Strasbourg (Strasbourg)

Bulgarian Historical Review/Revue bulgare d'Histoire (Sofija)

Bibliothèque de l'Information Grammaticale (Leuven/Louvain,

Belgium)

Bilge (Ankara)

Bilig. Türk dünyası sosyal bilimler dergisi /Journal of Social Sciences of the Turkish World (Ankara)

Bibliotheca Orientalis (Leiden, NL)

Bir (İstanbul)

Belgian Journal of Linguistics (Amsterdam)

British Journal of Middle Eastern Studies (London)

Bulletin of the John Rylands University Library of Manchester

(Manchester, UK)

Bedi Kartlisa (Paris)

Bodleian Library Record (Oxford)

Byzantine and Modern Greek Studies (Birmingham)

Beiträge zur Namensforschung (Heidelberg)

Bibliotheca Orientalis Hungarica (Budapest)

Bulgarian Past and Present (Sofija)

La Bretagne Linguistique. Cahiers du Groupe de Recherche sur

l'Économie Linguistique de la Bretagne (Brest)

Balkan Studies (Thessaloniki)

Bulletin de la Société de Linguistique de Paris (Paris)

British Society for Middle Eastern Studies. Bulletin (London)

Bulletin of the School of Oriental and African Studies (London)

Belgelerle Türk Tarihi Dergisi (Ankara)

Boğaziçi Üniversitesi Dergisi (İstanbul)

Boğaziçi Üniversitesi Degisi, Beşerî Bilimler/Boğaziçi University

Journal, Humanities (İstanbul)

Boğaziçi Üniversitesi Halkbilimi Yıllı̆̆ (İstanbul)

Bulletin i Universitetit Shtetëror të Tiranës. Seria shkencat shoqërore/

Bulletin of the State University of Tirana. Social sciences series (Tiranë)

Byzantinoslavica (Prague)

Central Asiatic Journal. International Periodical for the Languages,

Literature, History and Archeology of Central Asia (Wiesbaden) 


\begin{tabular}{|c|c|}
\hline CalWPL & Calgary Working Papers in Linguistics (University of Calgary) \\
\hline$C A S$ & Central Asian Survey (Oxford) \\
\hline$C B$ & Cahiers Balkaniques (Paris) \\
\hline$C E ́ B B L$ & Centre d'Études Balkaniques (Paris) \\
\hline CEMOTI & $\begin{array}{l}\text { Cahiers d'Études sur la Méditerranée Orientale et le Monde Turco- } \\
\text { Iranien (Paris) }\end{array}$ \\
\hline Çevren & Çevren (Priština) \\
\hline$C F$ & Cahiers de Fontenay (Fontenay-aux-Roses, France) \\
\hline CFS & $\begin{array}{l}\text { Cahiers Ferdinand de Saussure. Revue Suisse de Linguistique Générale. } \\
\text { (Genève, Switzerland) }\end{array}$ \\
\hline Choice & Choice (Middletown, CT) \\
\hline$C I L L$ & $\begin{array}{l}\text { Cahiers de l'Institut de Linguistique, Université Catholique de Louvain } \\
\text { (Louvain-la-Neuve, Belgium) }\end{array}$ \\
\hline CILT & $\begin{array}{l}\text { American Studies in the Theory and Linguistic Science IV: Current } \\
\text { Issues in Linguistic Theory (Amsterdam) }\end{array}$ \\
\hline CLing & Cercetări de Lingvistică (Cluj, Romania) \\
\hline$C L O$ & Cahiers Linguistiques d'Ottawa (Ottawa, Ontario) \\
\hline$C L S$ & $\begin{array}{l}\text { CLS ... Papers from the ... Annual Regional Meeting of the Chicago } \\
\text { Linguistic Society (Chicago, IL) }\end{array}$ \\
\hline CLTA & Cahiers de linguistique théorique et appliquée (Bucarest) \\
\hline$C O A$ & Cahiers d'Onomastique Arabe (Paris) \\
\hline CSLI & Center for the Study of Language and Information (Stanford University) \\
\hline$C S P$ & Canadian Slavonic Papers (Montreal) \\
\hline$C T$ & Cultura Turcica (Ankara) \\
\hline$C ̧ T D$ & Çağdaş Türk Dili (Ankara) \\
\hline$C ̧ T T A D$ & Çağdaş Türkiye Tarihi Araştırmaları Dergisi (İzmir) \\
\hline CUNY & City University of New York \\
\hline$C W P L$ & Cornell Working Papers in Linguistics (Ithaca, NY) \\
\hline$D A$ & Dilbilim Araştırmaları (Ankara) \\
\hline$D A b$ & $\begin{array}{l}\text { Dissertation Abstracts International. Abstracts of dissertations available } \\
\text { on microfilm or as xerographic reproductions. A. The Humanities and } \\
\text { Social Sciences (Ann Arbor, MI) }\end{array}$ \\
\hline Dacor & Dacoromania. Jahrbuch für östliche Latinität (Freiburg) \\
\hline$D A I$ & Dissertation Abstracts International (Ann Arbor, MI) \\
\hline$D A I-A$ & $\begin{array}{l}\text { Dissertation Abstracts International, Section A. The Humanities and } \\
\text { Social Sciences (Ann Arbor, MI) }\end{array}$ \\
\hline$D A I-B$ & $\begin{array}{l}\text { Dissertation Abstracts International, Section B. The Sciences and } \\
\text { Engineering (Ann Arbor, MI) }\end{array}$ \\
\hline$D A I-C$ & $\begin{array}{l}\text { Dissertation Abstracts International, Section C. Worldwide (Ann Arbor, } \\
\text { MI) }\end{array}$ \\
\hline$D d R$ & Doktorske disertacije Rezimei (Sarajevo) \\
\hline Dilbilim & $\begin{array}{l}\text { Dilbilim. İstanbul Üniversitesi Yabancı Diller Yüksek Okulu Fransızca } \\
\text { Bölümü dergisi /Linguistique. Revue du Département de français de } \\
\text { l’École Supérieure des langues étrangéres de l’Université d'Istanbul } \\
\text { (İstanbul) }\end{array}$ \\
\hline DilDe & Dil Dergisi (Ankara) \\
\hline DirHum & Dirāsāt-Humanities ('Ammān, Jordan) \\
\hline DIstGl & Dodatak Istorijskom Glasniku (Beograd) \\
\hline
\end{tabular}




\begin{tabular}{|c|c|}
\hline Diyalog & Diyalog. Interkulturelle Zeitschrift für Germanistik (Ankara) \\
\hline$D K M S$ & 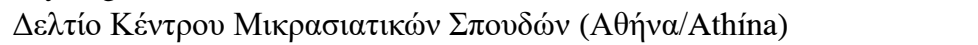 \\
\hline$D L$ & Deutsch lernen (Heidelberg) \\
\hline$D L Z$ & $\begin{array}{l}\text { Deutsche Literaturzeitung für Kritik der internationalen Wissenschaft } \\
\text { (Berlin) }\end{array}$ \\
\hline DMO Mitteilungen & Dokumentationsdienst Moderner Orient, Mitteilungen (Hamburg) \\
\hline$D o A$ & Doğu Akdeniz (Gazimağusa, Cyprus) \\
\hline$D o D$ & Doğu Dilleri (Ankara) \\
\hline$D \ddot{D}$ & Dil Öğretimi Dergisi (Ankara) \\
\hline$\Delta \omega \delta \omega \dot{v \eta}$ & 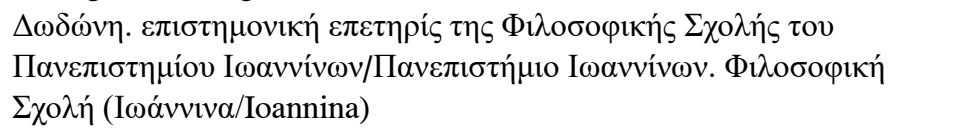 \\
\hline DoklSo & Doklady i Soobščenija. Institut Jazykoznanija AN SSSR (Moskva) \\
\hline$D R$ & Dacoromania (Cluj, Romania) \\
\hline DS-Nell & Dutch Studies published by NELL (Leiden) \\
\hline$D \ddot{U E F D}$ & Dicle Üniversitesi Eğitim Fakültesi Dergisi (Diyarbakır) \\
\hline DYaz & Dilbilim Yazıları (Ankara) \\
\hline$E A \ddot{U} E F D$ & Eskişehir Anadolu Üniversitesi Edebiyat Fakültesi Dergisi (Eskişehir) \\
\hline ÉBalk & Études Balkaniques (Sofija) \\
\hline$E B D E D$ & Ege Bat1 Dilleri ve Edebiyat1 Dergisi (Bornova, İzmir) \\
\hline Economist & The Economist (London) \\
\hline$E D$ & 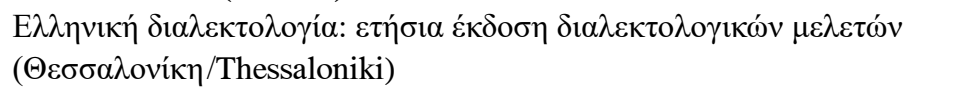 \\
\hline$E F A D$ & Edebiyat Fakültesi Araştırma Dergisi (Erzurum) \\
\hline$E L$ & Ezik i Literatura (Sofija) \\
\hline$E \lambda \lambda \eta v \imath \kappa \alpha ́$ & E $\lambda \lambda \eta v ı \kappa \alpha ́(\Theta \varepsilon \sigma \sigma \alpha \lambda o v i ́ \kappa \eta / T h e s s a l o n i k i)$ \\
\hline ÉMS & Études mongoles et sibériennes (Paris) \\
\hline Eothen & $\begin{array}{l}\text { Jahreshefte der Gesellschaft der Freunde Islamischer Kunst und Kultur } \\
\text { (München) }\end{array}$ \\
\hline Erasmus & $\begin{array}{l}\text { Erasmus. Speculum scientiarum. International Bulletin of Contemporary } \\
\text { Scholarship/Bulletin international de la science contemporaine } \\
\text { (Wiesbaden) }\end{array}$ \\
\hline Erdem & Erdem. İnsan ve Toplum Bilimleri Dergisi (Ankara) \\
\hline ESBAV & $\begin{array}{l}\text { Eğitim, Sağlık ve Bilimsel Araştırma Çalışmaları Vakfı (Anadolu } \\
\text { Üniversitesi, Eskişehir) }\end{array}$ \\
\hline ESCOL & $\begin{array}{l}\text { Proceedings of the ... Eastern states conference on linguistics (Cornell } \\
\text { University, Ithaca, NY) }\end{array}$ \\
\hline ÉSEE & $\begin{array}{l}\text { Études Slaves et Est-Européens/Slavic and East European Studies } \\
\text { (Montreal) }\end{array}$ \\
\hline EShq & Ethnografia Shqiptare (Tiranë) \\
\hline ESY & Eurasian Studies Yearbook (Indiana University, Bloomington, IN) \\
\hline Ethnology & Ethnology (Pittsburg, PA) \\
\hline Ethnopsychologie & Ethnopsychologie (Le Havre) \\
\hline Étimologija & $\begin{array}{l}\text { Ėtimologija. Rossijskaja Akademija Nauk, Institut Russkogo Jazyka } \\
\text { imeni V.V. Vinogradova (Moskva) }\end{array}$ \\
\hline Ë̈IFD & Erciyes Üniversitesi İlahiyat Fakültesi Dergisi (Kayseri) \\
\hline
\end{tabular}


EurH 21

$E \ddot{U} S B F D$

EÜTDEAD

$F D$

$F d L$

FEFAD

Filološki Pregled

FLang

FLet

FLing

FO

FoDo

FoL

FoLH

Forrás

Forum

FoSl

$\mathrm{FU}$

FUF

FVL

GADARBH

GASSF

GDAAD

$G D D$

Gengo

GGA

GJLL

GjuJ

GK

$G L$

GLECS

Glossa

Glotta

GLS

Gramma/TTT

GSU

GSU-KNF
Europäische Hochschulschriften / Publications universitaires européennes/European university studies. 21: Sprachwissenschaft /

Linguistique/Linguistics. (Frankfurt a.M.-Bern)

Ege Üniversitesi Sosyal Bilimler Fakültesi Dergisi (İzmir)

Ege Üniversitesi Edebiyat Fakültesi Türk Dili ve Edebiyatı Araştırmaları

Dergisi (İzmir)

Fonetică şi Dialectologie (Bucureşti)

Forum der Letteren (Den Haag)

Fen-Edebiyat Fakültesi Araştırma Dergisi (Erzurum)

Filološki Pregled (Beograd)

Foundations of Language (Dordrecht)

Forum der Letteren (Bedhouvedorp)

Folia Linguistica (The Hague)

Folia Orientalia (Kraków)

Folklora Doğru (İstanbul)

Folia Linguistica. Acta Societatis Linguisticae Europaeae (Berlin)

Folia Linguistica Historica. Acta Societatis Linguisticae Europaeae

(The Hague)

Forrás [=Kiskunság] (Kecskemét)

Forum. Yabancı sorunları üzerine araştırma dergisi (Frankfurt a.M.)

Folia Slavica (Colombus, $\mathrm{OH}$ )

Freie Universität Berlin

Finnisch-Ugrische Forschungen. Zeitschrift für finnisch-ugrische Sprach- und Volkskunde (Helsinki)

Forschungen zur Volks- und Landeskunde (Klausenburg-Hermannstadt)

Glasnik Arhiva i Društva arhivskih radnika Bosne i Hercegovine

(Sarajevo)

Gjurmime Albanologjike, Serie e Shkencave Filologjike (Prishtinë)

Güney-Doğu Avrupa Araştırmaları Dergisi (İstanbul)

Genel Dilbilim Dergisi (Ankara)

Gengo (Tōkyō)

Göttinger gelehrter Anzeiger (Göttingen)

The Georgetown Journal of Languages and Linguistics (Washington,

DC)

Gjuha Jonë (Tiranë)

Gengo Kenkyū. Journal of the Linguistic Society of Japan (Kyōto)

General Linguistics (University Park, PA)

Comptes rendus du Groupe Linguistique d'Études Chamito-Sémitiques (Paris)

Glossa (Burnaby, British Columbia)

Glotta (Göttingen)

Grazer Linguistische Studien (Graz)

Gramma/TTT. Tijdschrift voor taalwetenschap [continuation of Gramma \& TTT] (Dordrecht)

Godišnik na Sofijskija Universitet "Sv. Kliment Ochridski" (Sofija)

Godišnik na Sofijskija Universitet "Sv. Kliment Ochridski”, Fakultet po

klasičeski i novi filologii (Sofija) 


\begin{tabular}{|c|c|}
\hline$G S U-S F$ & $\begin{array}{l}\text { Godišnik na Sofijskija Universitet "Sv. Kliment Ochridski”, Fakultet po } \\
\text { slavjanski filologii. Problemi na bălgarskoto jezikoznanie/Annuaire de } \\
\text { l’Université de Sofia, Faculté des philologies slaves. Problèmes de la } \\
\text { linguistique bulgare (Sofija) }\end{array}$ \\
\hline$G S U-Z F$ & $\begin{array}{l}\text { Godišnik na Sofijskija Universitet "Sv. Kliment Ochridski", Fakultet po } \\
\text { zapadni filologii /Annuaire de l'Universitę de Sofia, Facultę des lettres } \\
\text { (Sofija) }\end{array}$ \\
\hline$G Z b$ & $\begin{array}{l}\text { Godišen Zbornik na Filozofskiot Fakultet na Univerzitetot vo Skopje/ } \\
\text { Annuaire de la Faculté de Philosophie de l'Université de Skopje } \\
\text { (Skopje) }\end{array}$ \\
\hline GZMBHS & Glasnik Zemaljskog Muzeja u Bosni i Hercegovini (Sarajevo) \\
\hline HAGEM & Halk Kültürlerini Araştırma ve Geliştirme Genel Müdürlüğü (Ankara) \\
\hline HandNFC & Handelingen van het ... Nederlands Filologencongress (Groningen) \\
\hline$H B H$ & Hacettepe Bulletin of Humanities (Ankara) \\
\hline$H E ́ L$ & Histoire, Épistémologie, Language (Lille) \\
\hline Hercegovina & Hercegovina (Mostar) \\
\hline$H F$ & Humanistisk forskning (Stockholm) \\
\hline$H J A S$ & Harvard Journal of Asiatic Studies (Cambridge, MA) \\
\hline HiRo & Hitotsubashi Ronsō (Tōkyō) \\
\hline Hist T & History Today (London) \\
\hline$H K \bar{A}$ & Ḥawlīyāt Kullīyat al-Ādāb bi-Ǧāmica cAyn Šams (al-Qāhira/Kairo) \\
\hline HК̈̈ & Halk Kültürü (İstanbul) \\
\hline$H P B$ & Das Historisch-Politische Buch (Göttingen) \\
\hline$H S B B D$ & Hacettepe Üniversitesi Sosyal ve Beşerî Bilimler Dergisi (Ankara) \\
\hline$H S p$ & Historische Spachforschung/Historical Linguistics (Göttingen) \\
\hline HSSS & Harvard Studies in Syntax and Semantics (Cambridge, MA) \\
\hline$H \ddot{U E F D}$ & Hacettepe Üniversitesi Edebiyat Fakültesi Dergisi (Ankara) \\
\hline$H \ddot{U} E \breve{g} F D$ & Hacettepe Üniversitesi Eğitim Fakültesi Dergisi (Ankara) \\
\hline$H \ddot{U} F D E B D$ & $\begin{array}{l}\text { Hacettepe Üniversitesi Fransız Dili ve Edebiyatı Bölümü Dergisi } \\
\text { (Ankara) }\end{array}$ \\
\hline HUS & Harvard Ukrainian Studies (Cambridge, MA) \\
\hline$H W P L$ & Harvard Working Papers in Linguistics (Cambridge, MA) \\
\hline$\dot{I} A r$ & İlmi Araştırmalar (İstanbul) \\
\hline$I B L A$ & Revue de l'Institut des Belles Lettres Arabes (Tunis) \\
\hline IC & Islamic Culture (Hyderabad) \\
\hline$\dot{I} D$ & İletişim Dergisi (İstanbul) \\
\hline$I F$ & Indogermanische Forschungen (Berlin) \\
\hline$\dot{I F M}$ & İstanbul Üniversitesi İktisat Fakültesi Mecmuası (İstanbul) \\
\hline$\ddot{I I F D}$ & İslâmî İlimler Fakültesi Dergisi (Ankara) \\
\hline$I J a$ & Inostrannye Jazyki. Sbornik statej (Moskva) \\
\hline$I J A L$ & International Journal of American Linguistics (Chicago, IL) \\
\hline IJCAS & International Journal of Central Asian Studies (Seoul) \\
\hline IJMES & International Journal of Middle East Studies (London-New York) \\
\hline IJSL & International Journal of the Sociology of Language (The Hague) \\
\hline IJTS & International Journal of Turkish Studies (Madison, WI) \\
\hline InfoDaF & Informationen Deutsch als Fremdsprache (München) \\
\hline INJAL & International Journal of Applied Linguistics (Oslo) \\
\hline $\operatorname{In} L$ & Incontri linguistici (Trieste) \\
\hline
\end{tabular}




\begin{tabular}{|c|c|}
\hline IP & Istoričeski Pregled (Sofija) \\
\hline$I P A J$ & Journal of the International Phonetic Association (Cambridge) \\
\hline Iran \& Caucasus & Iran and the Caucasus (Leiden) \\
\hline $\operatorname{IrodÚ} j$ & Irodalmi Újság/Gazette Littéraire Hongroise (Paris) \\
\hline Islam & $\begin{array}{l}\text { Der Islam. Journal of the History and Culture of the Middle East } \\
\text { (Berlin) }\end{array}$ \\
\hline IsMis & Islamska Misao (Sarajevo) \\
\hline$I s t \check{C}$ & Istorijski Časopis/Revue historique (Beograd) \\
\hline Istoriká & 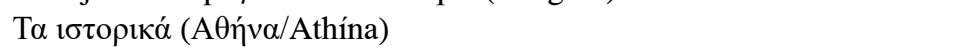 \\
\hline Ist $Z$ & Istorijski Zbornik (Banja Luka) \\
\hline İTED & İslam Tetkikleri Enstitüsü Dergisi (İstanbul) \\
\hline İTTT & Interdisciplinair Tijdschrift voor Taal- en Textwetenschap (Dordrecht) \\
\hline İÜ & İstanbul Üniversitesi \\
\hline İÜEF & İstanbul Üniversitesi Edebiyat Fakültesi \\
\hline İÜEFTDED & $\begin{array}{l}\text { İstanbul Üniversitesi Edebiyat Fakültesi Türk Dili ve Edebiyatı Dergisi } \\
\text { (İstanbul) }\end{array}$ \\
\hline$\dot{I} \ddot{U} F A D$ & İstanbul Üniversitesi Felsefe Arkivi Dergisi (İstanbul) \\
\hline IUPUAS & $\begin{array}{l}\text { Indiana University Publications, Uralic and Altaic Series (Bloomington, } \\
\text { IN-The Hague) }\end{array}$ \\
\hline$\dot{I} \ddot{U} S B D$ & İnönü Üniversitesi Sosyal Bilimler Dergisi (Malatya) \\
\hline İzlem & İzlem (Eskişehir) \\
\hline Izraz & Izraz (Sarajevo) \\
\hline$I z v A N A z L J a I$ & $\begin{array}{l}\text { Izvestija Akademii Nauk Azerbajdžanskoj SSR. Serija literatury, jazyka } \\
\text { i iskusstva (Baku) }\end{array}$ \\
\hline IzvANGrJaL & $\begin{array}{l}\text { Izvestija Akademii Nauk Gruzinskoj SSR. Serija jazyka i literatury } \\
\text { (Tbilisi) }\end{array}$ \\
\hline$I z v A N K a z F$ & $\begin{array}{l}\text { Izvestija Akademii Nauk Kazachskoj SSR, Serija filologičeskaja } \\
\text { (Alma-Ata) }\end{array}$ \\
\hline$I z v A N K a z O$ & $\begin{array}{l}\text { Izvestija Akademii Nauk Kazachskoj SSR, Serija obščestvennaja } \\
\text { (Alma-Ata/Almaty) }\end{array}$ \\
\hline$I z v A N L J a$ & Izvestija Akademii Nauk SSSR, Serija literatury i jazyka (Moskva) \\
\hline IzvANOLJa & $\begin{array}{l}\text { Izvestija Akademii Nauk SSSR, Seija otdelenie literatury i jazyka } \\
\text { (Moskva) }\end{array}$ \\
\hline$I z v A N O N$ & Izvestija Akademii Nauk SSSR. Serija obščestvennych nauk (Moskva) \\
\hline IzvANTurON & $\begin{array}{l}\text { Izvestija Akademii Nauk Turkmenskoj SSR, Serija obščestvennych nauk } \\
\text { (Ašchabad) }\end{array}$ \\
\hline$I z v I B E$ & Izvestija na Instituta za Bălgarski Ezik (Sofija) \\
\hline$I z v N B K M$ & Izvestija na Narodna Biblioteka "Kiril i Metodij" (Sofija) \\
\hline$J A$ & Journal Asiatique (Paris) \\
\hline$J A A S$ & $\begin{array}{l}\text { Journal of Asian and African Studies/Ajiya-Afurika gengo bunka } \\
\text { kenkyū (Tōkyō) }\end{array}$ \\
\hline$J A F$ & $\begin{array}{l}\text { The Journal of American Folklore (University of Illinois, Urbana- } \\
\text { Champaign, IL) }\end{array}$ \\
\hline$J A H$ & Journal of Asian History (Wiesbaden) \\
\hline JAOS & Journal of the American Oriental Society (New Haven, CT) \\
\hline JASA & Journal of the Acoustical Society of America (Providence, RI) \\
\hline$J b D a F$ & $\begin{array}{l}\text { Jahrbuch Deutsch als Fremdsprache. Intercultural German Studies } \\
\text { (München) }\end{array}$ \\
\hline
\end{tabular}




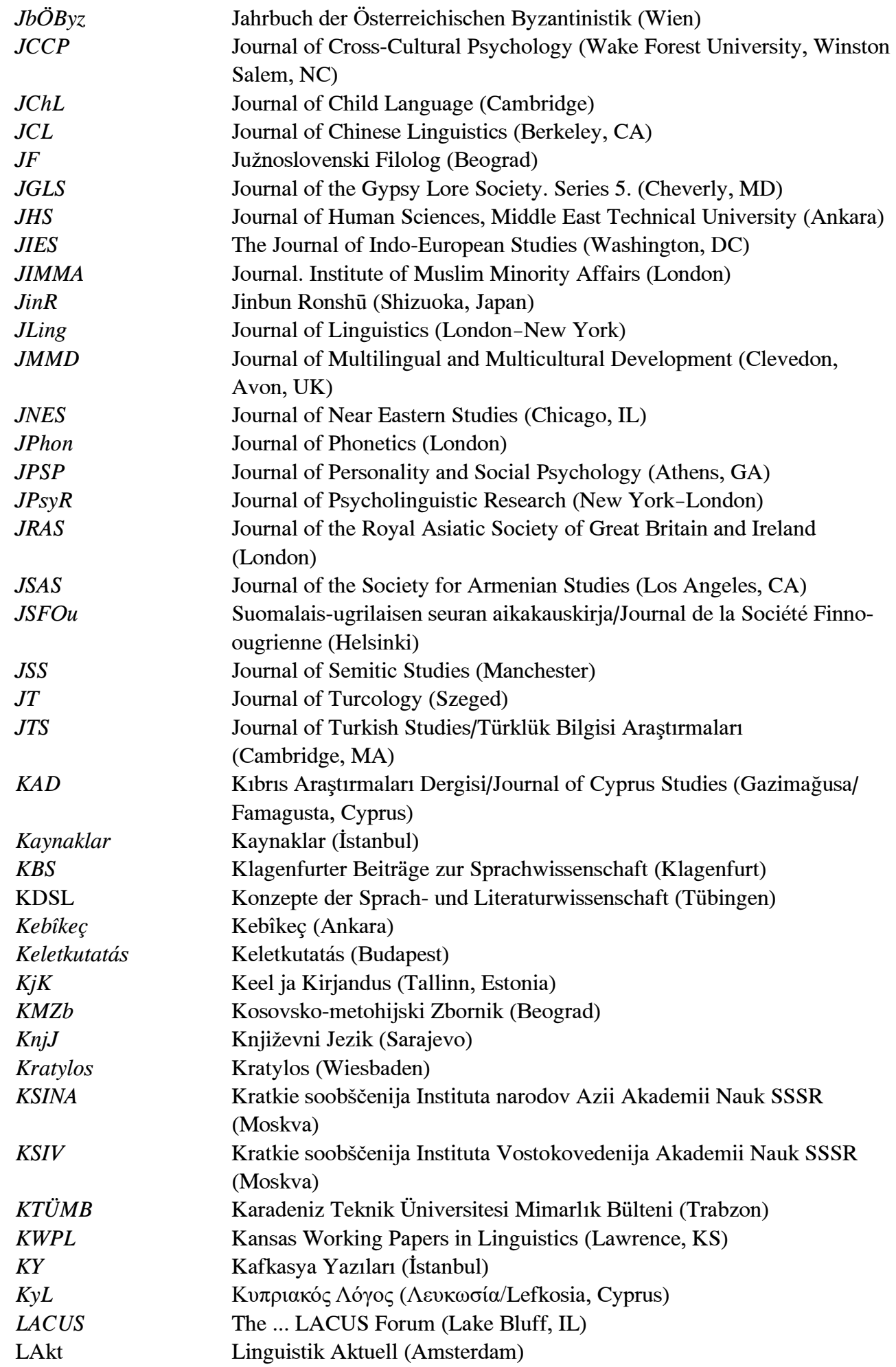


$L a L$

$L A n$

$L \& L C$

Language

LArb

LaSo

$L B$

LCC

$\mathrm{LeF}$

$\mathrm{Lg}$

$L I$

LingAt

Ling $B$

Lingua

Linguistica

Linguistics

Linguistique

LInt

Litera

$L L$

$L P L P$

$L R$

LRev

LSA

LSbIJa

LTWPL

MachT

MagySz

Makedonistika

MakF

MakJ

Maledicta

$M c G W P L$

MChr

MDTG

MEB

Medusa

MEJ

MESA Bulletin
Language Learning (Ann Arbor, MI)

Linguistica Antverpiensia (Antwerpen)

Literary and Linguistic Computing (Oxford)

Language (Baltimore, MD)

Linguistische Arbeiten (Berlin-New York-Tübingen)

Language et société (Paris)

Leuvense bijdragen. Tijdschrift voor Germaanse filologie (Leuven,

Belgium)

Language, Culture and Curriculum (Clevedon, Avon, UK)

Letterature di Frontiera (Roma)

Language. Journal of the Linguistic Society of America (Baltimore, MD)

Linguistic Inquiry (Cambridge, MA)

Linguistica Atlantica (Westminster, MD)

Linguistische Berichte (Hamburg)

Lingua. International Review of General Linguistics (Amsterdam)

Linguistica (Ljubljana)

Linguistics (Berlin)

La Linguistique. Revue de la Société Internationale de Linguistique

Fonctionnelle/Journal of the International Society for Functional

Linguistics (Paris)

Language International. The Magazine for the Language Professions

(Amsterdam)

Litera. İstanbul Üniversitesi Edebiyat Fakültesi İngiliz Filolojisi Dergisi

(İstanbul)

Limba şi Literatura (Bucharest/Bucureşti)

Language Problems and Language Planning (University of Texas,

Austin, TX)

Limba Romănă (Bucureşti)

The Linguistic Review (Dordrecht)

Linguistic Society of America (Washington, DC)

Leksikografičeskij Sbornik Instituta Jazykoznanija Akademii Nauk

SSSR (Moskva)

La Trobe Working Papers in Linguistics (Melbourne)

Machine Translation (Dordrecht, NL)

Magyar Szemle (Budapest)

Makedonistika (Skopje)

Makedonski Folklor (Skopje)

Makedonski Jazik (Skopje)

Maledicta. The International Journal of Verbal Aggression

(Waukesha, WI)

McGill Working Papers in Linguistics/Cahiers linguistiques de McGill

(Montreal, Québec)

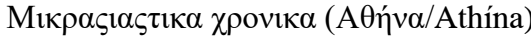

Mitteilungen der Deutsch-Türkischen Gesellschaft (Bonn)

Millî Eğitim Bakanlığı (Ankara)

Medusa. Svensk Tidskrift för Antiken (Stockholm)

The Middle East Journal (Washington, DC)

Middle East Studies Association Bulletin (New York) 


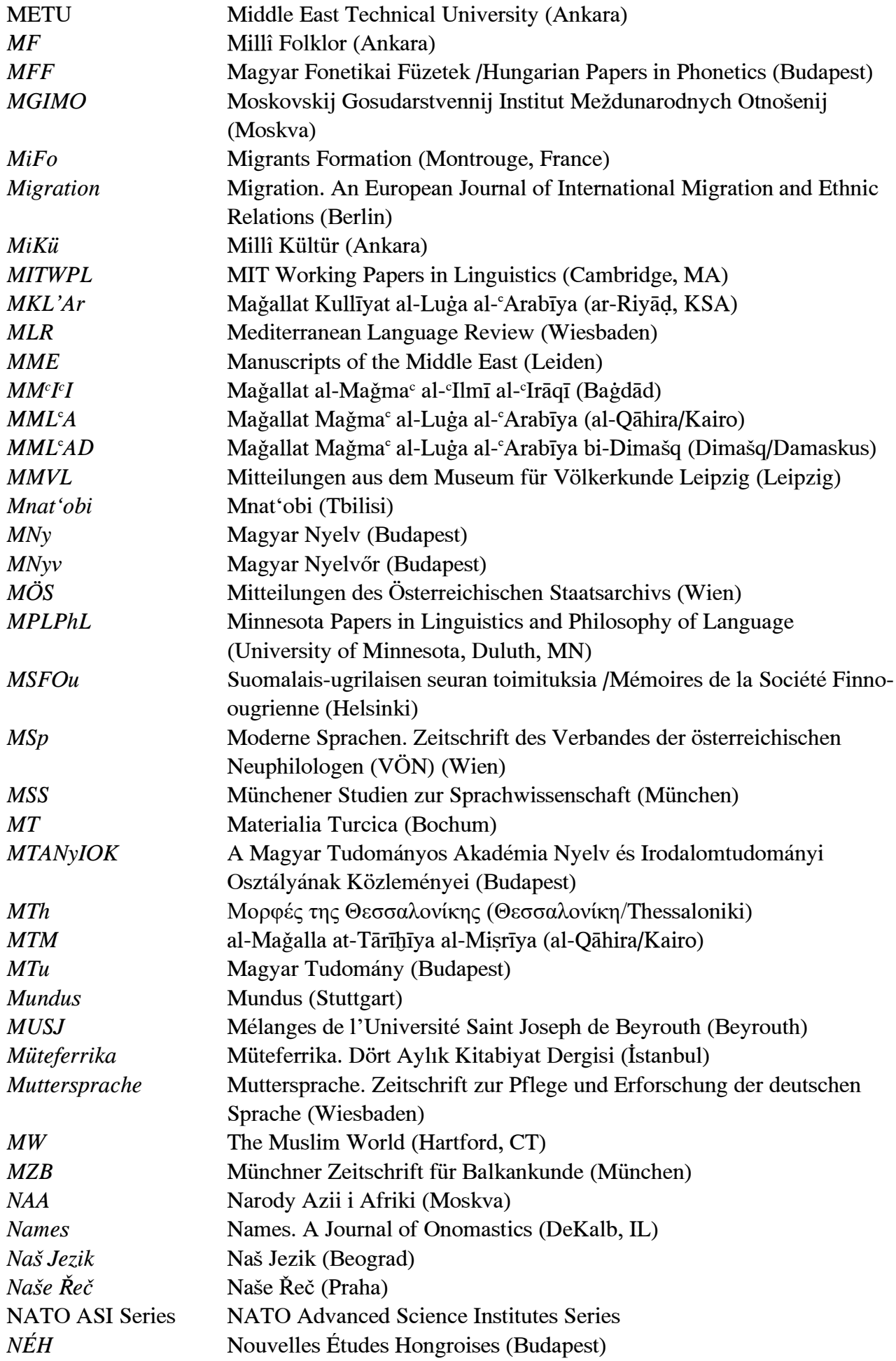




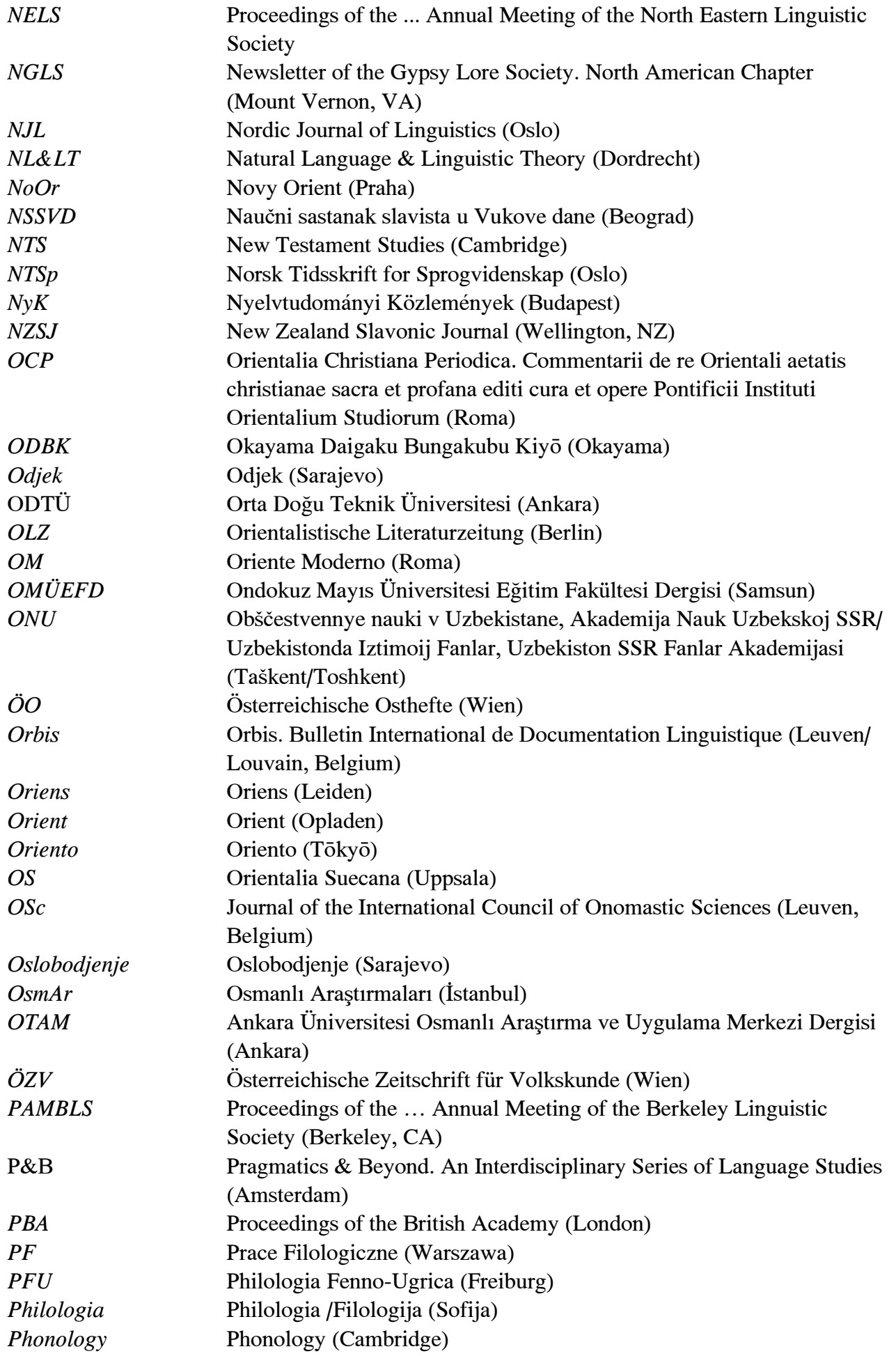




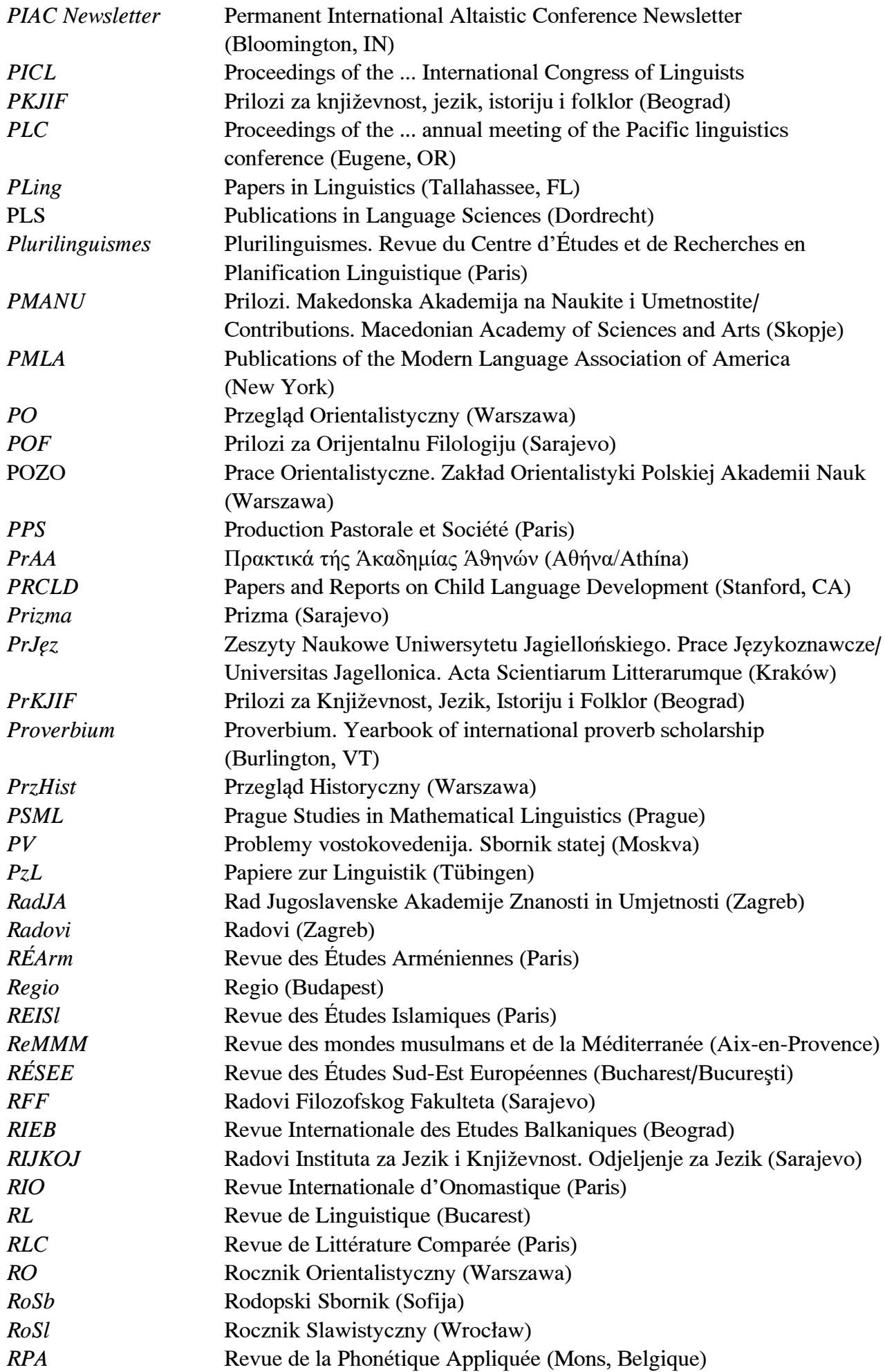




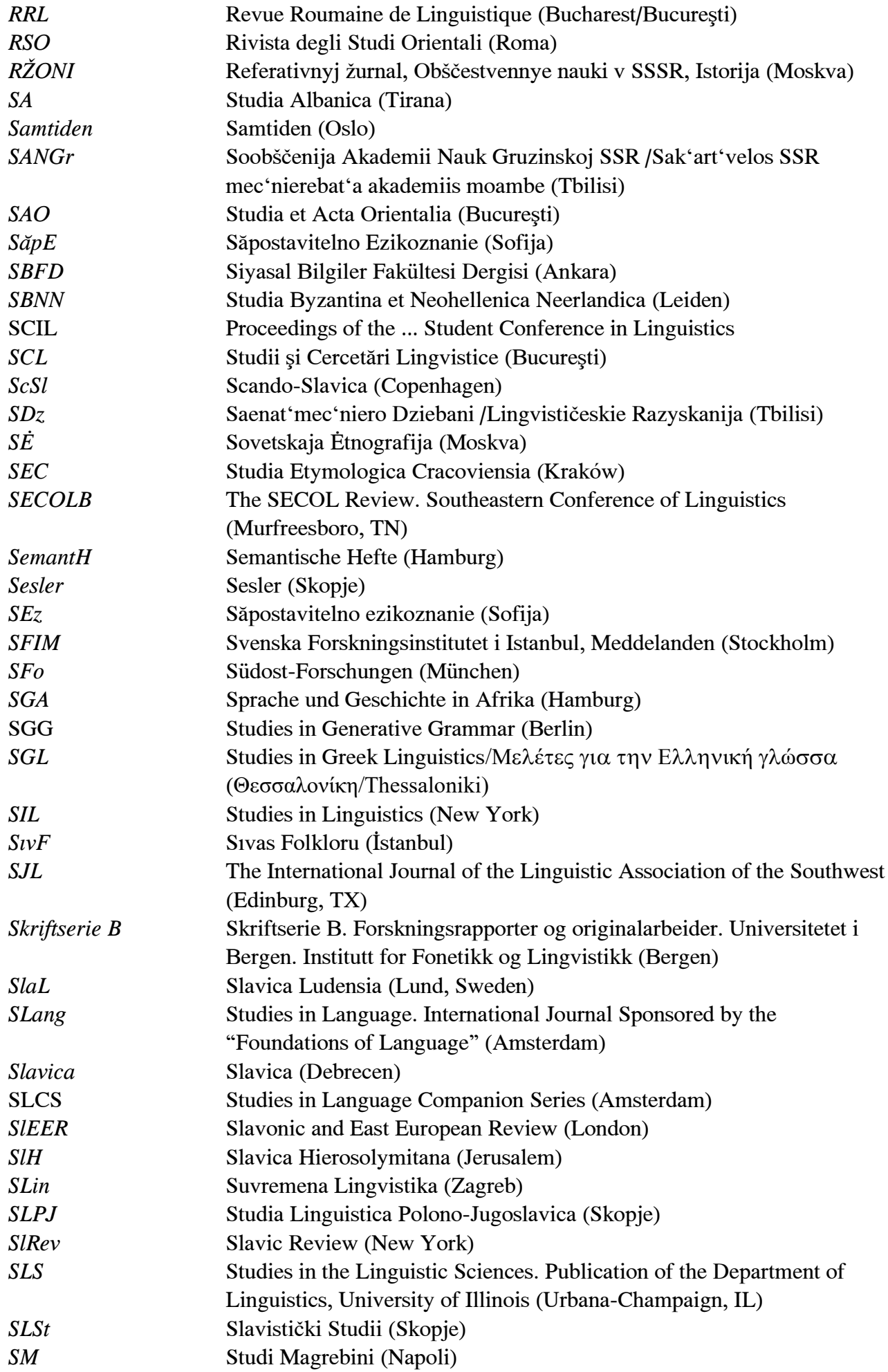


SNPh

$S O$

SOASWPLP

SoBi

SODV

SOEM

Spektator

Sprachdienst

SprOKrPAN

Šromebi

SSGL

SSH

SSLA

$S S p$

$S T$

STAR

STC

StF

STH

StOr

Studime

STUF

SuL

SV

SySem

Századok

SzÉ

TA

$T A D$

TaDe

TaMe

TASG News

TaTo

TBA

TBL

$T D$

$T D A$

$T D A D$

$T D A Y B$

$T D B$

$T D D$

TDe

TDGR
Studia Neophilologica (Stockholm)

Studia Orientalia (Helsinki)

School of Oriental and African Studies (SOAS) Working Papers in Linguistics and Phonetics (London)

Sosyal Bilimler (Elâzı̆̆

Südostdeutsche Vierteljahresblätter (München)

Südosteuropa Mitteilungen. Vierteljahresschrift der Südosteuropa-

Gesellschaft (München)

Spektator. Tijdschrift voor Neerlandistiek (Amsterdam)

Der Sprachdienst (Wiesbaden)

Polska Akademia Nauk, Oddział w Krakowie, Sprawozdania z

Posiedzeń Komisji (Wrocław)

Šromebi (Tbilisi)

Studies in Slavic and General Linguistics (Amsterdam)

Studia Slavica Hungarica (Budapest)

Studies in Second Language Acquisition (Cambridge)

Die slavischen Sprachen (Klagenfurt)

Sovetskaja Tjurkologija [cf. Tjurkologija] (Baku)

Studies on Turkish-Arab relations /Türk-Arab İlişkileri İncelemeleri

Y1llı̆̆ (Istanbul)

Studia Turcologica Cracoviensia (Kraków)

Studime Filologjike (Tiranë)

Studia Turco-Hungarica (Budapest)

Studia Orientalia (Helsinki)

Studime. Akademia e Shkencave dhe e arteve e Kosovës (Prishtinë)

Sprachtypologie und Universalienforschung (Berlin). Nachf. von ZPSK

Suvremena Lingvistika (Zagreb)

Soveckoe Vostokovedenie Akademii Nauk SSSR (Moskva)

Syntax and Semantics (New York)

Századok (Budapest)

Székesfehérvár Évszázadai (Székesfehérvár)

Turkologischer Anzeiger (Wien)

Tarih Araştırmaları Dergisi (Ankara)

Tarih Dergisi (İstanbul)

Tarih ve Medeniyet (İstanbul)

TASG News (London)

Tarih ve Toplum (İstanbul)

Türklük Bilimi Araştırmaları (Sivas)

Tübinger Beiträge zur Linguistik (Tübingen)

Türk Dili (Ankara)

Türk Dünyası Araştırmaları (İstanbul)

Türk Dünyası Araştırmaları Dergisi (İstanbul)

Türk Dili Araştırmaları Yıllığı-Belleten (Ankara)

Türk Dili Belleten (Ankara)

Türk Dili Dergisi (İstanbul)

Türkoloji Dergisi (Ankara)

Tōkyō daigaku gengogaku ronshū /Tokyo University Linguistics Papers (Tōkyō) 
TDiA

TDK

TE

TED

TEtD

TetrErg

Text

TF

TFA

TFAr

TFAYB

$T F B$

THAY

THKA

TIJaLTur

TIJaVJa

TILJNAzJa

TIPS

TIV

Tjurkologija

TK

TKA

TKAE

$T K D B$

$T L$

TLED

$T L F$

$T L P$

$T M$

TNWE

ToKe

Toplumbilim

ToTa

TPS

TRT

$T \check{S}$

$T S A B$

$T S t C$

TSU

TTbilU

TTbilUGN
Türk Dilleri Araştırmaları /Researches in Turkic Languages (Hacettepe Üniversitesi, Ankara)

Türk Dil Kurumu

Türk Edebiyatı (İstanbul)

Tarih Enstitüsü Dergisi (İstanbul)

Türk Etnografya Dergisi (Ankara)

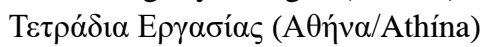

Text. An Interdisciplinary Journal for the Study of Discourse (Berlin)

Türk Folkloru (İstanbul)

Türk Folkloru Araştırmaları (Ankara)

Türk Folklor Araştırmaları. Aylık Halk Kültürü Dergisi (İstanbul)

Türk Folkloru Araştırmaları Yıllığı Belleten (Ankara)

Türk Folkloru Belleten (İstanbul)

Türk Halkbilim Araştırmaları Yıllı̆̆ı /Annual of Turkish Folklore

Researches (Ankara)

Türk Halk Kültürü Araştırmaları (Ankara)

Trudy Instituta Jazyka i Literatury Akademii Nauk Turkmenskoj SSR (Ašchabad)

Trudy Instituta Jazykoznanija Akademii Nauk Gruzinskoj SSR, Serija vostočnych jazykov (Tbilisi)

Trudy Instituta Literatury i Jazyka imeni Nizami Akademii Nauk

Azerbajdžanskoj SSR, Serija jazykoznanija (Baku)

Travaux de l'Institute de Phonétique de Strasbourg (Strasbourg)

Trudy Instituta Vostokovedenija Akademii Nauk Uzbekskoj SSR

(Taškent)

Tjurkologija [olim ST] (Baku)

Türk Kültürü (Ankara)

Türk Kültürü Araştırmaları (Ankara)

Türk Kültürünü Araştırma Enstitüsü (Ankara)

Türk Kütüphaneciler Derneği Bülteni (Ankara)

Turkic Languages (Wiesbaden)

Türk Lehçeleri ve Edebiyatı Dergisi (Ankara)

Texas Linguistic Forum (Austin, TX)

Travaux Linguistiques de Prague (Prague)

Türkiyat Mecmuası (İstanbul)

Turkish in North-West Europe (Tilburg, NL)

Toyoshi Kenkyū (Kyōto)

Toplumbilim (İstanbul)

Toplumsal Tarih (İstanbul)

Transactions of the Philological Society (Oxford)

Travaux et Recherches en Turquie (Paris-Louvain)

at-Turāta aš-Šac bī (Bağdād)

Turkish Studies Association Bulletin (Bloomington, IN)

Turkish Studies in Cyprus (Nicosia, Cyprus)

Trudy Samarkandskogo Gosudarstvennogo Universiteta (Samarkand)

Trudy Tbiliskogo Gosudarstvennogo Universiteta (Tbilisi)

Trudy Tbiliskogo Gosudarstvennogo Universiteta, Serija gumanitarnye nauki (Tbilisi) 
TTbilUJa

TTbilUV

TTK

TTT

TTWiA

TüAD

TüD

TULIP

Turcica

TVIIJa

TVUKM

TY

TYaz

$U A J b$ (N.F.)

UCLA

UCLAOPL

UJe

$U J b$

UK

$\ddot{U} l k \ddot{u}$

UporI

UÜIFD

$U Z A U$

UZAUV

UZJaL

UZKUF

$U Z L U$

UZLUV

UZTPI

VAN

Varia Eurasiatica

Varlık

VD

Vekove
Trudy Tbiliskogo Gosudarstvennogo Universiteta, Serija jazykoznanie (Tbilisi)

Trudy Tbiliskogo Gosudarstvennogo Universiteta, Serija

vostokovedenija (Tbilisi)

Türk Tarih Kurumu

Interdisciplinair Tijdschrift voor Taal- en Tekstwetenschap (Utrecht)

Toegepaste Taalwetenschap in Artikelen (Amsterdam)

Marmara Üniversitesi Fen-Edebiyat Fakültesi. Türklük Araştırmaları

Dergisi (İstanbul)

Türk Dünyası. Dil ve Edebiyat Dergisi (Ankara)

Turkish Linguistics Post (Mainz)

Turcica (Paris-Louvain)

Trudy Voennogo Instituta Inostrannych Jazykov (Moskva)

Trudove na Velikotărnovskija Universitet "Kiril i Metodij" (Veliko

Tărnovo)

Türk Yurdu

Türkiye Yazıları (Ankara)

Ural-Altaische Jahrbücher/Ural-Altaic Yearbook (Berlin-Bloomington-

London-Paris-Toronto; N.F. Wiesbaden)

University of California Los Angeles

University of California Los Angeles Occasional Papers in Linguistics

(Los Angeles)

Uporabno jezikolsovje (Ljubljana)

Ungarn-Jahrbuch (München)

Ulusal Kültür (Ankara)

Ülkü. Millî Kültür Dergisi (Ankara)

Uporedna Istraživanja (Beograd)

Uludağ Üniversitesi İlâhiyat Fakültesi Dergisi (Bursa)

Učenye Zapiski Azerbajdžanskogo Gosudarstvennogo Universiteta

(Baku)

Učenye Zapiski Azerbajdžanskogo Gosudarstvennogo Universiteta, Serija vostokovedenija (Baku)

Učenye Zapiski Azerbajdžanskogo Gosudarstvennogo Universiteta, Serija jazyka i literatury/Elmi Äsärlär, Dil vä ädäbijjat serijası (Baku/ Bak1)

Učenie Zapiski Kirgizskogo Gosudarstvennogo Universiteta

Filologičeskogo Fakul'teta (Biškek)

Učenye Zapiski Leningradskogo Gosudarstvennogo Universiteta A.A.

Ždanova (Leningrad)

Učenye Zapiski Leningradskogo Gosudarstvennogo Universiteta, Serija

vostokovedčeskich nauk (Leningrad)

Učenye Zapiski Taškentskogo Gosudarstvennogo Pedagogičeskogo

Instituta (Taškent)

Vestnik Akademii Nauk SSSR (Moskva)

Varia Eurasiatica (Szeged)

Varlık (İstanbul)

Vakıflar Dergisi (İstanbul)

Vekove (Sofija) 


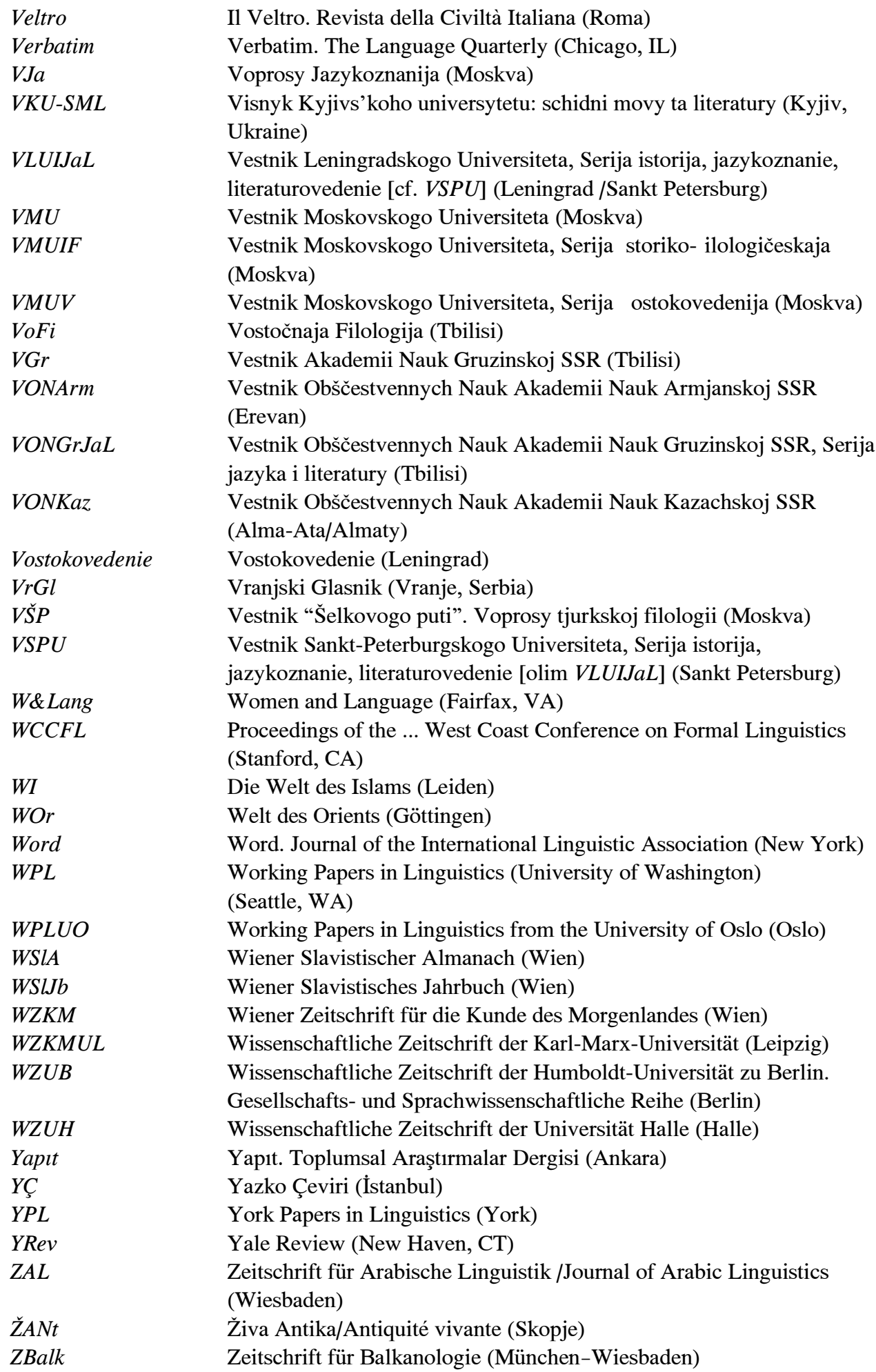


ZbFL

ZbRLL

$Z D L$

ZDMG

ZfE

ZFFP

ZFL

$Z G$

Život

ŽJ

$Z K V$

$Z L L$

$\mathrm{ZmaF}$

$Z M S F L$

$Z O$

ZPSK

$\mathrm{ZrP}$

$Z S D$

$Z S l$

$Z S P h$

ZSW

$\mathrm{AB}$

Abb.

Abt.

Afd.

alb.

App.

appr.

arab.

arm.

aserb.

Aufl.

Bd./Bde.

Bl.

Bosn. u. Herzeg.

bulg.

CA

ca.

cf.

$\mathrm{CH}$
Zbornik Matice Srpske za Filologiju i Lingvistiku (Novi Sad)

Zbornik radova Leksikografija i Leksikologija (Sarajevo)

Zeitschrift für Dialektologie und Linguistik. Beihefte (Wiesbaden)

Zeitschrift der Deutschen Morgenländischen Gesellschaft (Wiesbaden)

Zeitschrift für Ethnologie (Berlin)

Zbornik Filozofskog Fakulteta u Prištini/Buletin i punimeve shkencore të Fakultetit filozofik të Prishtinës (Prishtinë)

Zbornik za filologiju i lingvistiku /Archivum philologicum et linguisticum (Novi Sad, Serbia)

Zeitschrift für Geschichtswissenschaft (Berlin)

Život (Sarajevo)

Živi Jezici (Beograd)

Zapiski Kollegii Vostokovedov pri Aziatskom Muzeje (Leningrad)

Zeitschrift für Literaturwissenschaft und Linguistik (Frankfurt a. Main)

Zeitschrift für Mundartforschung (Wiesbaden)

Zbornik Matice Srpske za Filologiju i Lingvistiku (Novi Sad, Serbia)

Zeitschrift für Ostforschung. Länder und Völker im östlichen

Mitteleuropa (Marburg an der Lahn)

Zeitschrift für Phonetik, Sprachwissenschaft und Kommunikations-

forschung (Berlin)

Zeitschrift für romanische Philologie (Tübingen)

Zielsprache Deutsch (München)

Zeitschrift für Slavistik (Berlin)

Zeitschrift für slawische Philologie (Heidelberg)

Zeitschrift für Sprachwissenschaft (Göttingen)

\section{Sonstige Abkürzungen}

Alberta

Abbildung

Abteilung

Afdeling (Abteilung)

albanisch

Appendix (Anhang)

approximativ

arabisch

armenisch

Aserbaidschanisch

Auflage

Band/Bände

Blatt

Bosnien und Herzegowina

bulgarisch

California

cirka (um, etwa, ungefähr)

confer (vergleiche)

Confoederatio Helvetica, Schweiz 


\begin{tabular}{|c|c|}
\hline CT & Connecticut \\
\hline $\mathrm{DC}$ & District of Columbia \\
\hline d. H. & (im Jahr) der Hedschra \\
\hline Diss. & Dissertation \\
\hline ed(s). & editor, editors (Herausgeber) \\
\hline ELT & English Language Teaching \\
\hline engl. & englisch \\
\hline erw. & erweiterte \\
\hline et al. & et alii (und andere) \\
\hline Faks. & Faksimile \\
\hline fasc. & fasciculus (Heft) \\
\hline FL & Florida \\
\hline franz. & französisch \\
\hline GA & Georgia \\
\hline geb. & geboren \\
\hline georg. & georgisch \\
\hline gest. & gestorben \\
\hline holländ. & holländisch \\
\hline IL & Illinois \\
\hline IN & Indiana \\
\hline ital. & italienisch \\
\hline japan. & japanisch \\
\hline Jh. & Jahrhundert \\
\hline kasach. & kasachisch \\
\hline KS & Kansas \\
\hline KSA & Kingdom of Saudi-Arabia \\
\hline kungl. & kunglig (königlich) \\
\hline kyrill. & kyrillisch \\
\hline lat. & lateinisch \\
\hline Lfg. & Lieferung \\
\hline MA & Massachusetts \\
\hline $\begin{array}{l}\text { MA-Dissertation } \\
\text { mazed. }\end{array}$ & $\begin{array}{l}\text { Masters Dissertation (Magisterarbeit) } \\
\text { mazedonisch }\end{array}$ \\
\hline MD & Maryland \\
\hline MGr. & neugriechisch \\
\hline MI & Michigan \\
\hline MN & Minnesota \\
\hline M.S. & Master of Science \\
\hline Nachf. & Nachfolger \\
\hline $\mathrm{NC}$ & North Carolina \\
\hline N.F. & Neue Fassung \\
\hline NJ & New Jersey \\
\hline NL & Niederlande \\
\hline norweg. & norwegisch \\
\hline N.S. & Neue Serie, New Series, Nuova Serie \\
\hline NY & New York \\
\hline $\mathrm{NZ}$ & Neuseeland \\
\hline $\mathrm{OH}$ & Ohio \\
\hline
\end{tabular}




\begin{tabular}{|c|c|}
\hline $\begin{array}{l}\text { OR } \\
\text { osm. }\end{array}$ & $\begin{array}{l}\text { Oregon } \\
\text { osmanisch }\end{array}$ \\
\hline PA & Pennsylvania \\
\hline pers. & persisch \\
\hline Ph.D. & Doctor of Philosophy \\
\hline Phil. Diss. & Doktorarbeit, Dissertation (Phil. Fak.) \\
\hline q.v. & quod vide (siehe dort) \\
\hline Res. & Resümee \\
\hline Rez. & Rezension \\
\hline RI & Rhode Island \\
\hline rumän. & rumänisch \\
\hline russ. & russisch \\
\hline $\mathrm{S}$. & Seite \\
\hline s. & siehe \\
\hline s.a. & sine anno \\
\hline serbochorv. & serbo-kroatisch \\
\hline skr. & serbo-kroatisch \\
\hline s.l. & sine loco \\
\hline Sp. & Spalte \\
\hline span. & spanisch \\
\hline stlb. & stolbec (Spalte, columna) \\
\hline Taf. & Tafel \\
\hline T.C. & Türkiye Cumhuriyeti (Republik Türkei) \\
\hline techn. Berat. & technischer Berater \\
\hline TN & Tennessee \\
\hline tr. & traduit (übersetzt) \\
\hline türk. & türkisch \\
\hline TX & Texas \\
\hline u.a. & und andere \\
\hline überarb. & überarbeitet(e) \\
\hline UK & United Kingdom \\
\hline ung. & ungarisch \\
\hline $\begin{array}{l}\text { Univ. } \\
\text { un-numm. }\end{array}$ & $\begin{array}{l}\text { Universität, university } \\
\text { unnummeriert }\end{array}$ \\
\hline usw. & und so weiter \\
\hline VA & Virginia \\
\hline verbess. & verbesserte \\
\hline vs. & versus (contra, gegen) \\
\hline VT & Vermont \\
\hline WA & Washington State \\
\hline WI & Wisconsin \\
\hline z.B. & zum Beispiel \\
\hline zus. & zusammen \\
\hline
\end{tabular}


